\title{
Qualidade de Vida em Idosos com Distintos Níveis de Atividade Física
}

\author{
Quality of Life in Elderly Subjects with Different Levels \\ of Physical Activity
}

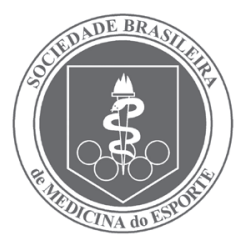

Artigo Original
José Jean de Oliveira Toscano' Antônio César Cabral de Oliveira²

1. Mestre em Ciências da Saúde; Núcleo de Pós-Graduação em Medicina - Departamento de Educação Física - Universidade Federal de Alagoas-UFAL.

2. Doutor em Ciência da Atividade Física e do Desporto;

Núcleo de Pós-Graduação em Medicina - Departamento de Educação Física - Universidade Federal de Sergipe.

Endereço para correspondência: Universidade Federal de Alagoas

- Curso de Educação Física.

Campus A.C. Simões, BR-04 - Norte, km 97, Cidade Universitária.

57072-970 - Maceió, AL.

E-mail: jjotoscano@yahoo.com.br

Submetido em 21/05/2007 Versão final recebida em 23/06/2008 Aceito em 24/10/2008

\section{RESUMO}

O estilo de vida saudável tem sido associado ao hábito de práticas de atividades físicas e, consequentemente, a melhores padrões de saúde e qualidade de vida. O principal objetivo deste estudo foi comparar a qualidade de vida em idosos com distintos níveis de atividade física. O tipo de estudo foi o transversal de base populacional e a amostra composta por 238 idosas, com media de idade de 69,2 $( \pm 6,6)$ anos, escolhidas aleatoriamente em 23 grupos de convivência do município de Aracaju-SE. O nível de atividade física foi avaliado pelo IPAQ (International Physical Activity Questionnaire) na sua versão longa e a qualidade de vida, por meio do SF-36 (The medical outcomes study 36-item short-form healthy survey). A análise estatística utilizou ferramentas da estatística descritiva e, para a comparação entre as variáveis, o teste de associação de Kruskal-Wallis com significância de $p \leq 0,05$. Foi identificada neste estudo diferença significativa entre o nível de atividade física e a qualidade de vida relacionada com a saúde. Mulheres idosas mais ativas apresentaram melhores resultados nos oito domínios da qualidade de vida investigados.

Palavras-chave: qualidade de vida, atividade física, envelhecimento.

\begin{abstract}
Healthy lifestyle has been associated with practice of physical activity and consequently with better health and quality of life standards. The aim of this study was to associate the levels of physical activity with the quality of life of the elderly. The study was transversal and a randomized sample was formed with 238 female elderly subjects ( $69.2 \pm 6.69$ years) drafted from 23 elderly groups of Aracaju. The level of physical activity was evaluated by IPAQ (International Physical Activity Questionnaire - long form) and quality of life by SF-36 (The medical outcomes study 36-item short-form healthy survey). Statistical analysis used descriptive statistics instruments and for analysis between variables Kruskal-Wallis test was used $(p<0.05)$. Significant association was identified in this study between the level of physical activity and health-related quality of life. Elderly women with high physical activity level presented better results in the eight health domains investigated.
\end{abstract}

Keywords: quality of life, physical activity, elderly.

\section{INTRODUÇÃO}

O novo quadro demográfico nacional e suas consequências epidemiológicas têm estimulado pesquisas não só para avaliar a saúde dos idosos, mas também permitindo otimizar os recursos humanos e financeiros para melhor subsidiar intervenções e implementações de programas de saúde para aquela população(1).

Nos últimos anos vem aumentando o interesse por estudos que relacionam a atividade física regular, sistemática, com o processo de envelhecimento saudável, principalmente quanto a seu impacto na qualidade de vida de idosos(2).

O conceito de qualidade de vida tem que ser compreendido como influenciado por todas as dimensões da vida e, assim, inclui, mas não deve estar limitado à existência ou não de morbidades. Contudo, diante do impacto que o conceito saúde pode ter na vida das pessoas, se fazem necessários indicadores que possam nortear operacionalmente essa dimensão da qualidade de vida. Assim, a qualidade de vida relacionada com a saúde refere-se não só à forma como as pessoas percebem seu estado geral de saúde, mas também o quão física, psicológica e socialmente estão na realização de suas atividades diárias(3).
Estudos de cunho epidemiológico envolvendo idosos são importantes para que seus resultados possam direcionar programas de promoção da saúde, principalmente no que diz respeito a comportamento mais ativo dessa população, que possa redundar em impactos positivos na qualidade de vida relacionada com a saúde. Trabalhos nessa perspectiva apontam que os adultos de mais idade são menos ativos que os adultos jovens e que as mulheres mais velhas são menos ativas que os homens mais velhos ${ }^{(4)}$.

Um estilo de vida saudável está associado ao incremento da prática de atividades físicas, sejam elas realizadas no âmbito do trabalho, da locomoção, do lazer e das atividades domésticas, e, como consequência, com melhores padrões de saúde e qualidade de vida.

A literatura aponta evidências epidemiológicas quanto ao efeito positivo de adoção de um estilo de vida ativo ou o envolvimento em programas de atividade física sistemática, na prevenção e minimização dos efeitos deletérios do envelhecimento ${ }^{(5)}$. O Brasil dispõe de poucos dados sobre a prevalência da atividade física e sua relação com a qualidade de vida entre pessoas idosas, principalmente do sexo feminino(6). 
Portanto, o estudo da qualidade de vida em idosos é um dos novos tempos, que possibilita contemplar envelhecimento com qualidade cotidiana, ultrapassando as oportunidades oferecidas meramente ao acaso ${ }^{(7)}$.

No contexto atual da assistência ao idoso, tem-se estimulado, por meio de iniciativas governamentais e não-governamentais, a adoção de estratégias de atenção a essa população. Entre elas está o atendimento em centros de convivência, onde são formados os grupos de convivência para idosos, forma aceita e difundida em todo o mundo, por apresentar respostas efetivas à problemática do idoso, que é o isolamento social(6).

A produção de conhecimento sobre os vários aspectos da qualidade de vida em idosos nas várias regiões do país deve ser um esforço cada vez mais crescente, para que assim se possa intervir de forma efetiva nos programas de política de atenção ao idoso.

Diante do exposto, o presente estudo teve como objetivo comparar a qualidade de vida em idosos com distintos níveis de atividade física.

\section{MÉTODOS}

O desenho escolhido para delinear o presente estudo foi o transversal. A população foi constituída por 587 mulheres, com idade mínima de 60 anos e cadastradas nos 23 grupos de convivência assistidos pela Secretaria Municipal de Assistência Social e Cidadania (Semasc) do município de Aracaju-SE. Esses grupos desenvolvem atividades de trabalho manual, leituras, palestras e ações terapêuticas.

Visando melhor representar a população a ser estudada, a amostra foi composta utilizando-se a técnica de seleção aleatória por estrato etário. A partir da população de referência, estimou-se que pelo menos 238 idosas deveriam participar do estudo, sendo estas distribuídas em cinco estratos etários, conforme se pode observar na tabela 1. Para dimensionar o tamanho da amostra foi utilizada a formula proposta por Barbeta ${ }^{(8)}$. A média de idade global das idosas foi de 69,2 $( \pm 6,6)$ anos. Após escolhida a amostra, foi determinado o nível de atividade física e a qualidade de vida das idosas.

Para determinar o nível de atividade física, foi empregada a versão longa do IPAQ (International Physical Activity Questionnaire), que classifica como "mais ativa" a pessoa que realiza atividade física em pelo menos 150 minutos por semana (min/sem), e"menos ativa" aquela com menos de $150 \mathrm{~min} / \mathrm{sem}^{(9)}$. Para utilização em pessoas idosas, foram feitas adaptações no referido instrumento(10).

Para avaliar a qualidade de vida das idosas foi empregado o instrumento SF-36 (The Medical Outcomes Study 36-item short-form healthy survey), traduzido e validado para a realidade brasileira por Ciconelli et al.(11). O SF-36 é um questionário multidimensional composto por 36 itens, com duas a seis possibilidades de respostas objetivas, distribuídos em oito domínios, que podem ser agrupados em dois grandes componentes: o físico (capacidade funcional, aspectos físicos, dor e estado geral de saúde) e o mental (saúde mental, vitalidade, aspectos sociais e aspectos emocionais).

Ambos os questionários foram aplicados na forma de entrevista. 0 centro de convivência era previamente comunicado sobre a visita, as idosas sorteadas para participarem da amostra eram convidadas pelos entrevistadores para locais no próprio centro que pudessem reservar o direito à privacidade de suas respostas; em todos os grupos foi possível concluir a coleta no mesmo dia da visita.

Um dos grupos de convivência foi escolhido aleatoriamente para a realização de um estudo-piloto, onde o roteiro de coleta foi testado pelo grupo de sete pesquisadores, objetivando padronizar os procedimentos. Os pesquisadores voltaram ao grupo de convivência na mesma semana para aplicar novamente os instrumentos; a concordância entre as duas aferições foi estimada em 0,76, considerada forte.
Todos os procedimentos de coleta de dados para este estudo foram realizados em um período de dois meses.

O presente trabalho também foi submetido e aprovado pelo comitê de ética em pesquisa em seres humanos da Universidade Federal de Sergipe (protocolo 0292004). Todas as participantes assinaram o termo de consentimento livre e esclarecido.

A análise estatística dos dados foi realizada utilizando-se os procedimentos da estatística descritiva (média, desvio padrão e distribuição de frequência). Como foi observado que os dados não obedeciam a um padrão de normalidade, a análise entre as variáveis foi efetuada utilizando-se o teste de associação não paramétrico de Kruskal-Wallis, admitindo-se o nível de significância de $p<0,05$.

\section{RESULTADOS}

As diversas formas de segmentação da população devem ser entendidas como uma primeira aproximação ao tema e elas estão apresentadas na tabela 1.

Tabela 1. Distribuição da amostra em relação às características sociodemográficas

\begin{tabular}{|c|c|c|}
\hline Características & $\mathbf{n}$ & $\%$ \\
\hline \multicolumn{3}{|l|}{ Estrato etário } \\
\hline $60-64$ & 69 & 29,0 \\
\hline $65-69$ & 67 & 28,2 \\
\hline 70-74 & 52 & 21,8 \\
\hline 75-79 & 30 & 12,6 \\
\hline$\geq 80$ & 20 & 8,4 \\
\hline \multicolumn{3}{|l|}{ Estado civil } \\
\hline Solteira & 30 & 12,6 \\
\hline Casada/vivendo com parceiro & 50 & 21,0 \\
\hline Divorciada/separada & 32 & 13,4 \\
\hline Viúva & 126 & 53,0 \\
\hline \multicolumn{3}{|l|}{ Escolaridade } \\
\hline Sem instrução & 75 & 31,5 \\
\hline 1 a 3 anos & 137 & 57,5 \\
\hline 4 a 7 anos & 18 & 7,5 \\
\hline 8 ou mais anos & 08 & 3,5 \\
\hline \multicolumn{3}{|l|}{ Religião } \\
\hline Católica & 225 & 94,5 \\
\hline Evangélica / Outras & 13 & 5,5 \\
\hline \multicolumn{3}{|l|}{ Trabalho } \\
\hline Remunerado & 16 & 6,7 \\
\hline Voluntário & 23 & 9,7 \\
\hline Não trabalha & 199 & 83,6 \\
\hline \multicolumn{3}{|l|}{ Rendimento pessoal mensal* } \\
\hline Sem rendimento & 24 & 10 \\
\hline Até 1 sal. min. & 128 & 53,8 \\
\hline Mais de 1 até 2 sal. min. & 64 & 26,9 \\
\hline Mais de 2 até 3 sal. min. & 10 & 4,2 \\
\hline Mais de 3 até 5 sal. min. & 05 & 2,1 \\
\hline Mais de 5 sal. min. & 07 & 3,0 \\
\hline
\end{tabular}

*Salário mínimo na época do estudo: Cr\$ 300,00

A tabela 2 apresenta a distribuição global das idosas em relação à realização de atividades físicas moderadas e vigorosas (períodos mínimos de 10 minutos contínuos) em cada domínio da atividade física.

Foi observado que a maior contribuição na demanda energética mensurada pelo IPAQ veio das atividades domésticas, seguidas das de lazer e de locomoção; foi pequena a contribuição das atividades físicas realizadas no trabalho. 
Tabela 2. Distribuição dos resultados da amostra nos diferentes tipos de atividade física

\begin{tabular}{c|c|c|c}
\hline Domínios do IPAQ & $\mathbf{n}$ & $\mathbf{X}(\mathbf{m i n} / \mathbf{s e m})$ & DP \\
\hline AF no trabalho & 14 & 90,7 & 80,9 \\
\hline AF no transporte & 170 & 60,6 & 42 \\
\hline AF doméstica & 199 & 178,0 & 159,1 \\
\hline AF lazer & 187 & 119,6 & 88,9 \\
\hline Tempo sentado & & & \\
Transporte & 135 & 118,7 & 104,9 \\
Durante a semana & 238 & 400,9 & 114,9 \\
Fim de semana & 238 & 410,1 & 115,7 \\
\hline
\end{tabular}

Considerando-se que os escores do SF-36 em cada domínio podem variar de zero a 100 e, que quanto maior o valor, melhor a qualidade de vida relacionada com a saúde, os resultados mostram que os domínios que apresentaram melhores escores foram: vitalidade, aspectos físicos, aspectos emocionais e aspectos sociais. Os de menor resultado foram os de saúde mental, capacidade funcional, dor e estado geral de saúde (tabela 3).

Tabela 3. Distribuição dos resultados da amostra nos domínios da QVRS

\begin{tabular}{l|c|c}
\hline \multicolumn{1}{c|}{ Domínios } & Média & Desvio Padrão \\
\hline Capacidade Funcional & 58,9 & 23,0 \\
\hline Aspectos Físicos & 67,4 & 37,8 \\
\hline Dor & 59,9 & 24,7 \\
\hline Estado Geral de Saúde & 61,9 & 21,3 \\
\hline Vitalidade & 67,2 & 18,7 \\
\hline Aspectos Sociais & 80,5 & 23,5 \\
\hline Aspectos Emocionais & 75,1 & 37,9 \\
\hline Saúde Mental & 54,9 & 7,7 \\
\hline
\end{tabular}

Ao classificar-se a amostra segundo o nível de atividade física, foi encontrado um contingente de $65 \%$ de idosas categorizadas como "mais ativas" e, consequentemente, $35 \%$ foram enquadradas como "menos ativas".

Ao comparar-se os diversos domínios da qualidade de vida entre os grupos de idosas mais ativas com as menos ativas, pode-se verificar que, em todos os domínios, os resultados dos indivíduos mais ativos foram significativamente superiores.

\section{DISCUSSÃO}

As características da população idosa do presente estudo apresentam um perfil menos favorecido em relação aos aspectos socioeconômicos. Provavelmente, os resultados reflitam as condições investigadas, particularmente de idosos mais pobres, os quais frequentam mais intensamente os grupos de convivência.

As atividades físicas realizadas dentro e ao redor da habitação de forma moderada a vigorosa (atividade física doméstica) representaram o maior gasto energético das idosas em relação aos outros três domínios; as tarefas relatadas mais comuns foram lavar pratos, lavar banheiro, varrer a casa, passar pano e/ou lavar a casa. De acordo com Ainsworth ${ }^{(12)}$, a demanda energética das mulheres durante sua vida é representada principalmente palas atividades domésticas e estima-se que estas despendam 3,9 horas por dia em trabalhos domésticos e em tarefas de cuidados com a família.

Dentre as mais ativas, 33\% realizavam atividades físicas de lazer. Essas ações representaram o segundo domínio de atividade física com maior dispêndio energético entre as idosas. Trabalhos de Yusuf et al. ${ }^{(13)} \mathrm{e}$ Mazo ${ }^{(6)}$ apontam que a caminhada, a ginástica e a natação constituem as principais atividades físicas das mulheres idosas.

No presente estudo foi observado que as atividades físicas de lazer eram realizadas em grande parte no próprio grupo de convivência, utilizando-se dos exercícios de alongamento e ginástica localizada que são orientados por estagiários de Educação Física. Contudo, foi observado que essas atividades não despertam grande interesse, sendo verificada pouca adesão, exceto em dois grupos que oferecem dança em dias específicos.

Nas atividades físicas de transporte, todas as idosas fazem uso da caminhada como meio de locomoção, atingindo média de 60,6 min/sem. Em estudo de Mazo ${ }^{(6)}$ foi encontrada média de 114 min/sem para a caminhada como forma de deslocamento em idosas. A proposta nesse sentido é de que as idosas possam ser estimuladas a realizar deslocamentos de forma mais intensa, bem como utilizar percursos mais longos.

As atividades físicas de trabalho foram as que menos contribuíram com o dispêndio energético, fato já esperado, uma vez que aproximadamente 84\% das avaliadas não desempenhavam atividades laborais.

As idosas que fizeram parte deste estudo passam, durante a semana, grande parte do dia na posição sentada e tempo ainda maior nos fins de semana. Há também muito tempo despendido naquela posição em meios de transporte. Estudos de Andresen e Meyers ${ }^{(14)}$ apontaram que as principais atividades detectadas no dia a dia de mulheres idosas foram ver televisão, ouvir rádio, costurar, ler e receber visitas. Essas atividades são predominantemente de intensidade leve, adotam em geral postura sentada e não promovem nenhum estímulo benéfico à saúde.

De acordo com o IBGE ${ }^{(15)}$, no Brasil, ver televisão é o maior componente na ocupação do tempo livre das pessoas. A falta de atividade

Tabela 4. Comparação dos domínios da QVRS com os distintos níveis de atividade física

\begin{tabular}{|c|c|c|c|c|c|c|c|c|}
\hline Níveis de AF & $\begin{array}{c}C F \\
X \text { DP }\end{array}$ & $\begin{array}{c}\text { AF } \\
X \text { DP }\end{array}$ & $\begin{array}{l}\text { DOR } \\
X \text { DP }\end{array}$ & $\begin{array}{r}\text { EGS } \\
X \text { DP }\end{array}$ & $\begin{array}{l}\text { VITAL } \\
\text { X DP }\end{array}$ & $\begin{array}{c}\text { AS } \\
X \text { DP }\end{array}$ & $\begin{array}{c}A E \\
X D P\end{array}$ & $\begin{array}{r}S M \\
X \quad D P\end{array}$ \\
\hline $\begin{array}{l}\text { Mais ativo } \\
\text { Menos ativo }\end{array}$ & $\begin{array}{l}70,1 \pm 20,1 \\
46,9 \pm 23,4\end{array}$ & $\begin{array}{l}75,3 \pm 35,4 \\
52,9 \pm 37,9\end{array}$ & $\begin{array}{l}64,1 \pm 23,1 \\
52,2 \pm 26,0\end{array}$ & $\begin{array}{l}69,1 \pm 18,4 \\
48,8 \pm 20,1\end{array}$ & $\begin{array}{l}72,9 \pm 16,0 \\
56,9 \pm 18,9\end{array}$ & $\begin{array}{l}85,1 \pm 22,6 \\
72,5 \pm 23,0\end{array}$ & $\begin{array}{l}81,6 \pm 33,4 \\
63,5 \pm 42,9\end{array}$ & $\begin{array}{l}56,6 \pm 7,3 \\
51,8 \pm 7,7\end{array}$ \\
\hline$p$ & 0,000 & 0,000 & 0,000 & 0,000 & 0,000 & 0,000 & 0,001 & 0,000 \\
\hline
\end{tabular}

$\mathrm{CF}=$ Capacidade funcional; $\mathrm{AF}=$ Aspectos físicos; $\mathrm{DOR}=$ Dor; $\mathrm{EGS}=$ Estado geral de saúde; $\mathrm{VITA}=$ Vitalidade; $\mathrm{AS}=\mathrm{Aspectos}$ sociais; $\mathrm{AE}=\mathrm{Aspectos}$ emocionais; $\mathrm{SM}=\mathrm{Saúde}$ mental. 
física e a quantidade de horas vendo televisão estão associadas significativamente aos marcadores bioquímicos de obesidade e de risco de doenças cardiovasculares ${ }^{(16)}$.

Considerando a condição de saúde crônica do idoso, nem o indicador de morbidade, nem os cuidados formais em serviços de saúde podem impactar a qualidade de vida daquela população. Sendo assim, o instrumento de qualidade de vida pode ser um indicador importante na avaliação das intervenções de promoção da saúde em idosos.

Destacam-se neste estudo os escores obtidos no domínio "aspectos sociais". Farquhar ${ }^{(17)}$ identificou que os contatos sociais entre os idosos demonstram ser componentes valiosos para boa qualidade de vida quanto ao status de saúde.

A avaliação da gravidade e relevância de um problema de saúde em mulheres idosas parece determinada mais pela possibilidade de enfrentá-lo do que pelo problema em si, e este pode ser exacerbado ou minimizado, pela inexistência ou existência de suporte familiar ou comunitário, o que aponta para a existência de associações entre autoavaliação da saúde e rede social de apoio(18).

Apesar de as relações sociais dos idosos geralmente acontecerem no meio familiar, quando os mesmos participam de grupos de convivência, as relações se estendem a seus pares dentro do grupo, aumentando assim o suporte social.

Na diversidade inerente à qualidade de vida há um elemento básico e interligado com todos os outros, que é a capacidade para realizar movimentos corporais de forma eficiente. A qualidade de vida pode sofrer baixa considerável, caso essa possibilidade de realização motora esteja limitada. As dificuldades na locomoção, no manuseio de instrumentos ou na manutenção e adaptação de posturas nas diferentes tarefas do cotidiano competem para a diminuição da autonomia do indivíduo, principalmente no idoso, com consequências previsíveis para sua qualidade de vida(19).

Como já foi comentado no presente estudo, nos oito domínios testados pelo SF-36, as idosas que possuíam melhor nível de atividade física registraram melhores resultados que as de pior nível de atividade física.

Baseados em extensa revisão de pesquisas que associam atividade física relacionada com qualidade de vida e saúde, Rejeski et al. ${ }^{(20,}$ e Rejeski e Mihalko(21) apresentaram evidências indiretas da influência da atividade física sobre a qualidade de vida relacionada com a saúde, indicando efeitos positivos do exercício sobre sintomas ligados tanto aos domínios da qualidade de vida no âmbito da saúde física, quanto aos da saúde mental. Na revisão dos referidos autores há elevado número de trabalhos estabelecendo relações positivas do estilo de vida ativo com as atividades da vida diária e o domínio das funções física, cognitiva e social. Essa percepção positiva pode ser exemplificada por meio da redução na dor e na fadiga, no vigor e energia, no desempenho cognitivo e neuropsicológico, nos estados de tensão e ansiedade, além de efetiva redução do estado de depressão, melhoria da atividade mental e relacionamento social.

Quando o foco é saúde física, em um recente estudo populacional realizado na Austrália com mais de oito mil idosos a partir de 65 anos, verificou-se que o nível de atividade física está associado com a melhoria dos aspectos físicos ${ }^{(22)}$.

A falta de atividade física regular é um potencial fator de risco que pode aumentar o declínio funcional e os custos com tratamentos. Nesse sentido, o incremento de programas de atividade física pode ser empregado como estratégia eficaz nos efeitos da dor, no aumento da energia, vitalidade, capacidade funcional, dentre outros aspectos físicos ${ }^{(5,23)}$.
Em se tratando especificamente da saúde mental, em uma sistemática revisão de estudos, Taylor et al. ${ }^{(24)}$ concluíram que os efeitos são claros quanto ao bem-estar psicológico provocado pela prática da atividade física em idosos, sendo um dos mecanismos de explicação para esses efeitos o aumento do fluxo cerebral durante o exercício. Algumas evidências sugerem que a atividade física sistemática também pode estar associada com a prevenção ou o atraso no desenvolvimento da demência ou do mal de Alzheimer ${ }^{(25)}$.

Faz-se importante destacar que os efeitos da atividade física, tanto na saúde física quanto na mental, frequentemente apareceram independentemente do desenho do estudo, idade, gênero, nacionalidade ou tipo da intervenção.

Estudos com metodologia semelhante, dentro de um contexto sociocultural, que possam aproximar os resultados sobre a relação da atividade física com a qualidade de vida relacionada com a saúde em mulheres idosas e de meia-idade, foram realizados nos últimos anos no Sul do Brasil ${ }^{(6,26)}$. Os achados apresentam diferença significativa entre os níveis de atividade física; as idosas mais ativas foram as que apresentaram médias mais elevadas nos domínios físico e psicológico. E, também, indivíduos insuficientemente ativos, quando comparados com os ativos, tinham probabilidade menor de apresentar nível positivo de qualidade de vida.

Na cidade do Porto, Portugal, idosos que praticavam atividade física apresentavam valores médios mais elevados e estatisticamente diferentes nas oito dimensões do SF-36, em relação ao grupo de indivíduos que não o faziam ${ }^{(27)}$. Em estudo também na Europa, com população idosa representativa da Espanha, foi observado que idosos envolvidos em atividades físicas apresentaram escores mais altos em praticamente todos os domínios do SF-36(28).

De modo específico, programas de atividade física podem contribuir de forma expressiva na qualidade de vida na população de idosos, tanto pelo engajamento social que eles promovem, quanto pelo estímulo positivo nos aspectos físicos, o que na prática resulta em maior autonomia. Os níveis elevados de atividade física parecem intervir de maneira positiva pelo fato de estarem associados às limitações funcionais que interferem direta ou indiretamente em todos os domínios da qualidade de vida relacionada com a saúde ${ }^{(29)}$.

Enfim, há consenso de que a atividade física pode intervir de forma diferente nos vários domínios da qualidade de vida em mulheres idosas e que a relação do nível de atividade física com o aumento da percepção da qualidade de vida relacionada com a saúde se dá de forma diretamente proporcional|(30,31).

Assim sendo, um processo de intervenção nessa realidade, no sentido de aumentar o nível de atividade física das idosas menos ativas e também ampliando a possibilidade de demanda energética nos aspectos físicos pouco explorados por aquelas classificadas como mais ativas, parece relevante.

Os grupos de convivência podem representar uma estratégia eficaz de política pública de promoção de um estilo de vida ativo. Foi verificado que, para ser fisicamente ativo, o convívio social e a integração em grupos são um forte indicador ${ }^{(32)}$.

O importante é que as ações voltadas para a atividade física não tenham o intuito apenas de ocupar o tempo livre do idoso ou de tratá-lo como pessoa incapaz. É preciso evitar o estabelecimento de estruturas infantilizadoras. Apenas ofertar atividade física nos grupos de convivência não é suficiente. Programas nesse sentido devem ser precedidos de informação sobre seus benefícios, avaliação das condições, necessidades e expectativas dessa população em relação a uma mudança de comportamento. 
Por fim, cabe ressaltar que o número de pesquisas em idosos no Brasil, utilizando os instrumentos empregados no presente estudo, ainda é reduzido, o que dificulta uma discussão mais ampliada e destaca a originalidade do manuscrito.

\section{CONCLUSÃO}

Diante dos dados observados, conclui-se que a qualidade de vida de idosas que possuem maior nível de atividade física é melhor que as de menor nível de atividade física.
Sendo assim, é importante que haja um planejamento de ações voltadas para esse grupo populacional, que é crescente, no sentido de preparar a sociedade brasileira para um envelhecimento mais saudável, o que aumentará a qualidade de vida adicional adquirida ao longo de décadas.

Todos os autores declararam não haver qualquer potencial conflito de interesses referente a este artigo.

\section{REFERÊNCIAS BIBLIOGRÁFICAS}

1. Testa MA, Simonson DC. Assessment of quality of life outcomes. N Engl J Med 1996,334:835-40.

2. Paffenbarger RS, Lee I. Physical activity and fitness for health and longevity. Res Q Exerc Sport 1996;67:11-28.

3. MMWR. Health-related quality of life measures United States - 1993. Atlanta: Center for Diseasses Control and Prevention, 1995;44:195-200

4. Kamimoto LA, Easton AN, Maurice E. Surveillance for live health risks among older adults - United States, 1993-1997. MMWR, 1999;48:89-156.

5. Nelson ME, Rejeski WJ, Blair SN, Duncan PW, Judge JO, King AC, Macera CA, Castaneda-Sceppa C. Physical activity and public health in older adults: recommendation from the American College of Sports Medicine and the American Heart Association. Med Sci Sports Exerc 2007;39:1435-45.

6. Mazo, GZ. Atividade física e qualidade de vida de mulheres idosas. [Tese de doutorado]. Porto, Faculdade de Ciências do Desporto e de Educação Física da Universidade do Porto; 2003.

7. Cardoso V, Gonçalves LHT. Qualidade de vida de pacientes geriátricos ambulatoriais portadores de multipatologias crônicas. Rev. Cien. Saúde, Florianópolis, 1998;17:148-64.

8. Barbeta, PA. Estatística aplicada às ciências sociais. $4^{a}$ ed. Florianópolis: Editora UFSC, 2001

9. Marshall A, Bauman A. The international physical activity questionnaire: summary report of reliability e validity studies. DRAFT IPAQ summary, 2001:1-25.

10. Benedetti TB, Mazo GZ, Barros MVG. Aplicação do Questionário Internacional de Atividades Físicas para avaliação do nível de atividades físicas de mulheres idosas: validade concorrente e reprodutibilidade teste-reteste. R Bras Ci e Mov 2004;12:25-34

11. Ciconelli RM, Ferraz MB, Santos W, Meinão I. Quaresma MR. Tradução para a língua portuguesa e validação do questionário genérico de avaliação da qualidade de vida SF-36 (Brasil SF-36) Rev Bras Reumatol 1999:39:143-50

12. Ainsworth B. Issues in the assessment of physical activity in woman. Res Q Exerc Sport 2000;71:37-42.

13. Yusuf HR, Croft JB, Giles WH, Anda RF, Casper ML, Caspersen CJ, et al. Leisure-time physical activity among older adults. Arch Internal Med 1996;156:1321-26.

14. Andresen EM, Meyers AR. Health-related quality of life outcomes measures. Arc Phys Med Rehabil 2000;81:30-44.

15. IBGE. Coordenação de população e indicadores sociais, Rio de Janeiro: IBGE, 2004

16. Teresa TF, Frank BH, Jie Y, Nain-Feng C, Donna S, Geoffrey HT, et al. Leisure-time physical activity, television watching, and plasma biomarkers of obesity and cardiovascular disease risk. Am J Epidem 2000;152:1171-8.

17. Farquhar, M. Elderly people's definitions of quality of life. Soc Sci Med 1995;41:1439-46
18. Grundy E, Sloggett A. Health inequilities in the older population: the role of personal capital, social resources and socio-economic circumstances. Soc Sci Med 2003;56:935-47.

19. Nahas MV. Atividade física, Saúde e Qualidade de vida: conceitos e sugestões para um estilo de vida ativo. $2^{\mathrm{a}}$ ed. Londrina: Midiograf, 2001.

20. Rejeski WJ, Brawley LR, Shumaker SA. Physical activity and health-related quality of life. Exerc Sport Sci Rev 1996;24:71-108.

21. Rejeski WJ, Mihalko SL. Physical activity and Quality of life in older adults. Gerontol A Biol Science Med Science 2001;56:23-35

22. Lim K, Taylor L. Factors associated with physical activity among older people - a population based study. Prev Med 2005;40:33-40.

23. Stiggelbout M, Popkema D Y, Hopman-Rock M, de Greef M, van Mechelen W. Once a week is not enough: effects of a widely implemented group based exercise programme for older adults. A randomized controlled trial. J Epidemiol Community Health 2004;58:83-8.

24. Taylor AH, Cable NT, Faulkner G, Hillsdon M, Narici M, Van Der Bij AK. Physical activity and older adults: a review of health benefits and the effectiveness of interventions. J Sports Sci 2004;22:703-25.

25. Landi F, Russo A, Bernabei R. Physical activity and behavior in the elderly: a pilot study. Arch Gerontol Geriatr Suppl 2004;9:235-41.

26. Silva DK. Atividade física habitual e qualidade de vida relacionada à saúde de mulheres com doença vascular periférica. [Dissertação de Mestrado] Florianópolis, Centro de Desportos da Universidade Federal de Santa Catarina, 2002.

27. Ribeiro JLS. A influência da actividade física, na qualidade de vida relacionada com a saúde, em indivíduos com mais de 65 anos. [Dissertação de Mestrado] Porto, Faculdade de Ciências do Desporto e de Educação Física da Universidade do Porto, 2002.

28. Guallar-Castillon P, Santa-Olalla PP, Banegas JR, Lopez E, Rodriguez-Artalejo F. Physical activity and quality of life in older adults in Spain. Med Clin 2004;123:606-10.

29. Azpiazu GM, Cruz JA, Villagrasa F JR, Abanades HJC, Garcia MN, Valero de Bernabe FA. Factors related to perceived poor health condition or poor quality of life among those over 65 years of age. Rev. Esp Salud Public. 2002;76:683-99.

30. Koltyn, KF. The association between physical activity and quality of life in older women. Women's Health Issues 2001;11:471-80.

31. Brown DW, Brown DR, Heath GW, Balluz L, Giles WH, Ford ES, et al. Associations between physical activity dose and health-related quality life. Med Sci Sports Exerc 2004;36:890-6.

32. Stahl T, Rutten A, Nutbeam D, Bauman S, Kannas L, Abel G, et al. The importance of the social environment for physically active lifestile-results from an international study. Social Sci Med 2001;52:1-10. 